\title{
Successful adaptation during COVID-19 pandemic: oropharyngeal cancer biopsies under local anaesthetic
}

\author{
Fergus Cooper ${ }^{1}$, Panagiotis Asimakopoulos ${ }^{1}$, Kim Ah-See $^{1}$, and Muhammad Shakeel ${ }^{1}$ \\ ${ }^{1}$ Aberdeen Royal Infirmary
}

October 12,2020

\begin{abstract}
Key points: 1. In selected patients, biopsies of oropharyngeal lesions including the tonsils can be performed under local anaesthetic safely. 2. The technique described was found to be well tolerated by the patients and there were no adverse outcomes. 3. Performing these biopsies under local anaesthetic eliminates the morbidity associated with general anaesthesia. 4. Performing these biopsies under local anaesthetic increases operating room availability which is particularly important in the current economic climate and considering the covid-19 pandemic. 5. Performing these biopsies under local anaesthetic is thought to reduce the time from initial consultation to reaching a decision regarding treatment.
\end{abstract}

Dear Sir/Madam,

In response to an advice statement from the Scottish Health Technologies Group (SHTG) asking clinicians to consider utilisation of outpatient biopsy for the prompt and cost effective diagnosis of head and neck cancer [1], we would like to share our successful recent local experience.

We have a short case series of patients who had a head and neck lesion amenable to biopsy under local anaesthetic. Our rate of performing these biopsies has increased further since the covid-19 pandemic and the subsequent reduced availability of operating theatres. Cases were identified prospectively as any patient who had a lesion in the pharynx amenable to biopsy under local anaesthetic. The STROBE reporting guidance was used. The table below (Table 1) demonstrates 18 cases of patients with suspicious pharyngeal lesions where we have performed local anaesthetic biopsy. We also present the turnaround times between initial consultation and performing the biopsy, and from the biopsy to the histological findings. All cases of malignancy were discussed in the Head and Neck multidisciplinary team meeting within 7 days of the result.

The technique we employ for local anaesthetic biopsies of the oropharynx involves the patient sitting on a reclining chair with head support. The ENT surgeon should have a good head light and suction available. Lidocaine or Lidocaine with Phenylephrine throat spray is applied topically to the area to be biopsied and the area is then infiltrated with 2-4mls of Lidocaine $2 \%$ with 1:80,000 Adrenaline via a dental needle and syringe, using a Lack tongue depressor if required. If the area being biopsied is a tonsil, the medial exophytic portion is targeted. A $6 \mathrm{~mm}$ punch is used along with Blakesley forceps and disposable scissors to excise multiple pieces of the lesion for biopsy (Figure 1). We have found that once anaesthetised, the tonsil can be held with the Blakesley forceps and retracted medially and superiorly without significant distress to the patient. In our experience, the bleeding is controllable with $6 \%$ hydrogen peroxide gargles (5mls diluted in $20 \mathrm{mls}$ water) and suction. We have not had to utilise bipolar forceps for haemostasis for these biopsies, however, this is available in our clinic area, just in case.

We have found this method of biopsy to be safe in selected patients and beneficial by avoiding the risks of general anaesthesia. It is also cost and resource effective by saving precious operating theatre time as well 
as being in the patient's best interest owing to the reduction in time from first clinical contact to treatment decision.

We advocate the use of this technique within Otolaryngology departments with a similar setup for selected patients.

Key points:

1. In selected patients, biopsies of oropharyngeal lesions including the tonsils can be performed under local anaesthetic safely.

2. The technique described was found to be well tolerated by the patients and there were no adverse outcomes.

3. Performing these biopsies under local anaesthetic eliminates the morbidity associated with general anaesthesia.

4. Performing these biopsies under local anaesthetic increases operating room availability which is particularly important in the current economic climate and considering the covid-19 pandemic.

5. Performing these biopsies under local anaesthetic is thought to reduce the time from initial consultation to reaching a decision regarding treatment.

Table 1: Characteristics of study group

( $\mathrm{SCC}=$ squamous cell carcinoma; $\mathrm{R}=$ Right; $\mathrm{L}=$ Left $)$

\begin{tabular}{lllll}
\hline Number & Age of patient & Site of lesion & Time from initial consultation to biopsy (days) & Diagnosis from biopsy \\
\hline 1 & 51 & Tonsil (R) & 0 & P16 positive poorly diff \\
2 & 58 & Tonsil (L) & 0 & P16 positive poorly diff \\
3 & 39 & Tonsil (L) & 25 & P16 positive poorly diff \\
4 & 77 & Tonsil (L) & 18 & P16 negative SCC in sit \\
5 & 73 & Tonsil (L) & 23 & P16 positive poorly diff \\
6 & 66 & Tonsil (L) & 19 & P16 negative moderatel \\
7 & 66 & Tonsil (R) & 0 & P16 positive poorly diff \\
8 & 55 & Tonsil (L) & 1 & HPV positive moderate \\
9 & 57 & Tonsil (L) & 0 & P16 negative poorly diff \\
10 & 85 & Tonsil (L) & 7 & B-cell non Hodgkin Lyn \\
11 & 63 & Tonsil (L) & 0 & Benign tonsil tissue \\
12 & 79 & Tonsil (R) & 2 & P16 negative moderatel \\
13 & 68 & Soft palate (R) & 1 & moderately differentiate \\
14 & 60 & Nose (L) & 10 & moderately differentiate \\
15 & 71 & Nose (L) & 0 & moderately differentiate \\
16 & 52 & Postnasal space (L) & 43 & undifferentiated non-ker \\
17 & 60 & Floor of mouth (L) & 0 & Moderately differentiate \\
18 & 61 & Floor of mouth (L) & 0 & Moderately differentiate \\
\hline
\end{tabular}

Fig. 1 Exophytic tumour involving the left oropharynx. A punched out area (white arrow) could be seen where a $6 \mathrm{~mm}$ biopsy punch was used to take the biopsy under local anaesthetic in the clinic 


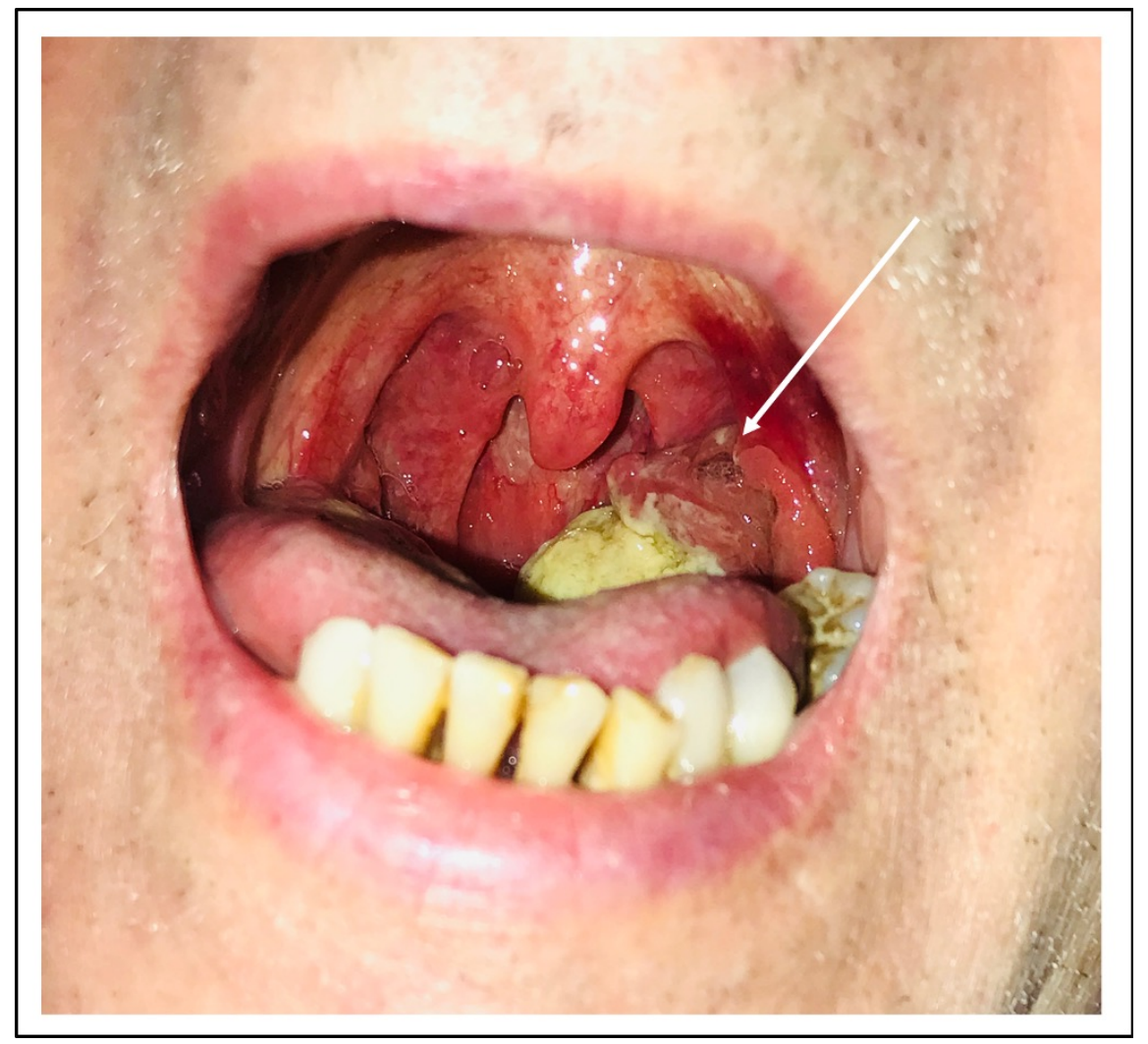

\section{References}

SHTG Advice statement: Outpatient biopsy, for diagnosis of suspicious lesions of the larynx, pharynx and tongue base. Healthcare Improvement Scotland, October 2018. Advice statement 012-18.

\section{Data Availability Statement}

The data that support the findings of this study are available on request from the corresponding author. The data are not publicly available due to privacy or ethical restrictions. 


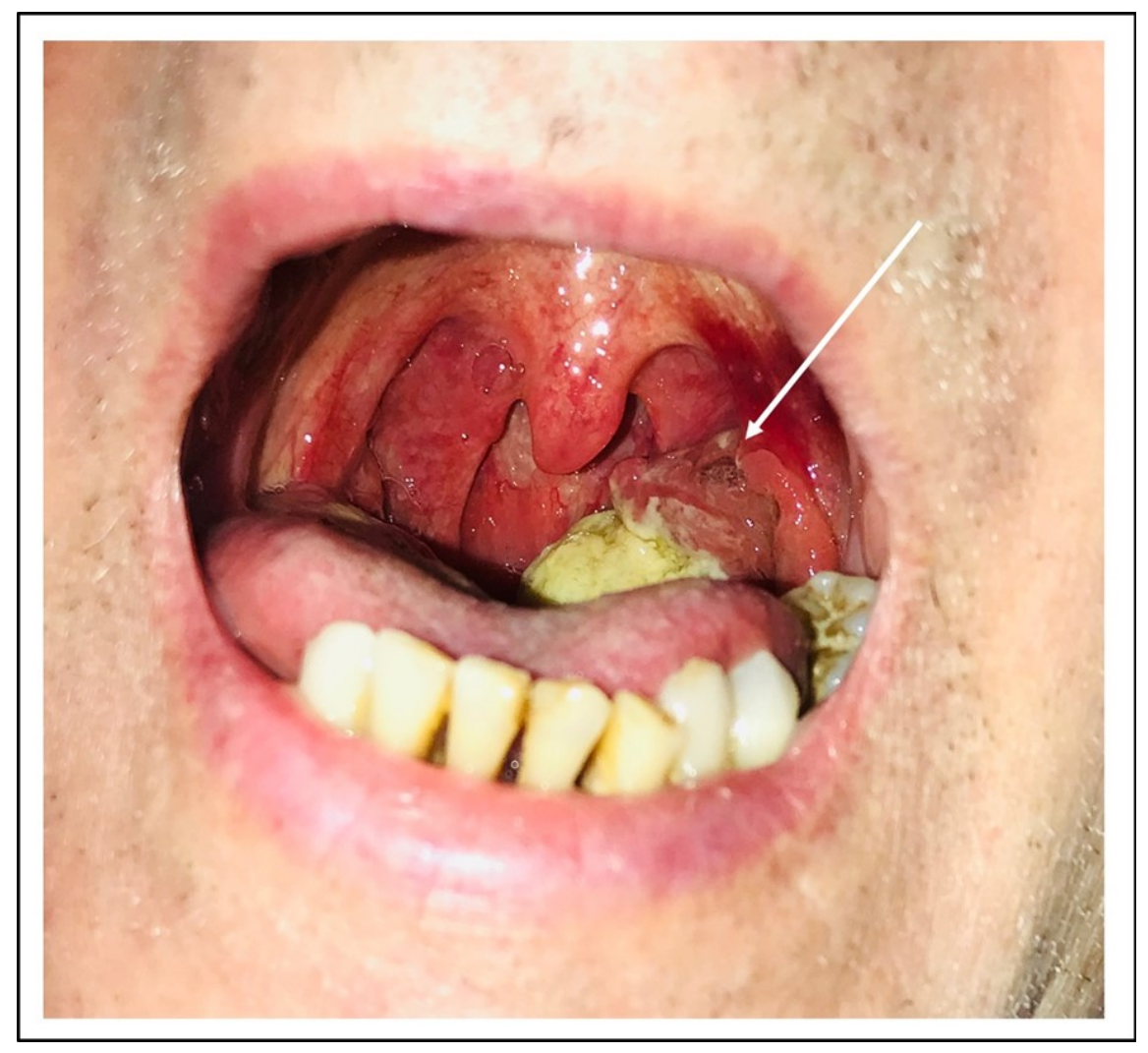

